\title{
Exploring the Subspace Method for Network-Wide Anomaly Diagnosis
}

\author{
Anukool Lakhina, Mark Crovella, Christophe Diot \\ \{anukool, crovella\}ecs.bu.edu, christophe.dioteintel.com
}

Categories and Subject Descriptors:

C.2.3 [Computer-Communication Networks]: Network Operations

General Terms: Measurement, Performance, Security

Keywords: Anomaly Detection, Whole-Network Analysis

\section{INTRODUCTION}

Network-wide anomalies represent unusual operating conditions that may span multiple links. These anomalies are important to detect from an operational standpoint because they impact multiple routers and can cause disruptive congestion in the network. The central problem with diagnosing network-wide anomalies is that their cause can vary considerably, from DoS attacks to bugs to configuration errors.

Recently, we introduced an important subset of network-wide anomalies called volume anomalies [2]. Volume Anomalies are unexpected changes that occur in the origin destination (OD) flow traffic of a network. They are important because they capture changes in the network's traffic matrix. In [2], we developed the subspace method to detect, identify and quantify volume anomalies from simple SNMP-based link bytecounts.

In this poster, we build on our earlier work and apply subspace methods directly to OD flow traffic (instead of link traffic). We first show how to extend the subspace method in this context and also to three different traffic data types: \# of bytes, \# of packets and \# of IP flows. We then present initial results on the anomalies we found by analyzing four weeks of OD flow traffic data collected from the Abilene Internet 2 backbone network. Our complete results can be found in [1].

\section{EXTENDING THE SUBSPACE METHOD}

In the context of OD flows, anomaly diagnosis using the subspace framework has the following three components: detecting the time of an anomaly, identifying the OD flows exhibiting the anomaly at that time, and finally, quantifying the amount of missing or additional traffic in the anomaly.

The subspace method examines the traffic in all OD flows simultaneously as a multivariate timeseries. It then exploits the low intrinsic dimensionality of OD flows [3], and separates the set of OD flows into disjoint subspaces corresponding to normal and anomalous traffic patterns. The normal subspace captures patterns of diurnal traffic behavior and the anomalous subspace captures sharp changes in traffic state. Once these subspaces have been constructed, the set of OD flow traffic can be decomposed into modeled and

Copyright is held by the author/owner

SIGCOMM'04 Workshops, Aug. 30 \& Sept. 3, 2004, Portland, Oregon, USA. ACM 1-58113-942-X/04/0008. residual components, by projecting the OD flows on each subspace.

Detection requires one to inspect the residual traffic for points in time when unusual events occur. To identify the OD flows exhibiting an anomaly at the detected time, we find the smallest set of OD flows that collectively account for the detected spike in the residual traffic, and mark these as the anomalous OD flows. Quantifying the size of the identified OD flow anomaly is straightforward and can be estimated as the difference between the modeled and true traffic.

As in [2], the subspace method effectively detected anomalies with a very low false alarm rate, even in this new context.

\section{PRELIMINARY RESULTS}

We apply the subspace method on OD flow traffic, defined as the \# of bytes, \# of packets and \# of IP flows. We find that each data type captures a unique aspect of a traffic anomaly. For instance, a spike in the \# of packets in an OD flow may not translate to a corresponding spike in the \# of bytes for that OD flow, if the packet sizes are small (e.g. TCP SYN packets). Similarly, spoofed DoS attacks would cause a spike in the \# of unique IP flows, but not necessarily in the \# of bytes.

We also find that multiple OD flows can exhibit an anomaly at the same time. By examining the origin and destination PoP for the anomalous OD flows, we can learn more about the anomaly. For instance, we find cases where a set of OD flows had a common destination PoP, and all exhibited spikes in the \# of IP flows constituting them, suggesting a DDoS attack on a customer. More sophisticated hypotheses can be formulated by accounting for the size of the spike (positive or negative) in the anomalous OD flows. For example, when two OD flows share the same destination and one has a positive spike while the other exhibits a negative spike, then this indicates a network-wide traffic shift. Egress load balancing or changes in BGP policy are likely candidate explanations for this phenomenon. As another example, network failure or outage events can manifest as a set of OD flows, each with a negative traffic spike.

By coupling the three types of OD flow traffic with their topological structure, we classified a broad set of anomalies, ranging from DoS attacks, flash crowds, port scanning, worm propagation, downstream traffic engineering, and network outage, as detailed in [1].

\section{REFERENCES}

[1] A. Lakhina, M. Crovella, and C. Diot. Characterization of Network-Wide Anomalies in Traffic Flows. In ACM SIGCOMM Internet Measurement Conference, Taormina, Italy, October 2004.

[2] A. Lakhina, M. Crovella, and C. Diot. Diagnosing Network-Wide Traffic Anomalies. In ACM SIGCOMM, Portland, August 2004.

[3] A. Lakhina, K. Papagiannaki, M. Crovella, C. Diot, E. D. Kolaczyk, and N. Taft. Structural Analysis of Network Traffic Flows. In ACM SIGMETRICS, New York, June 2004. 